\section{Immunological needles in the gene therapy haystack: applying a genetic paradigm to gene therapy

\author{
PR Lowenstein
}

Gene Therapy (2004) 11, 1-3. doi:10.1038/sj.gt.3302186

"I cannot promise to teach you all geology, I can only fire your imaginations." Adam Sedgwick; In: JW Clark and T Mc Hughes, The Life and Letters of Adam Sedgwick, 2 vols, Cambridge: Cambridge University Press, 1890, vol 2, p. 489; cited by Janet Browne in Charles Darwin, Voyaging, 1995, p. 139.

Replication-competent and replication-defective viral vectors have been developed as therapeutic options in cancer, infectious disorders, degenerative, metabolic, and genetic diseases. Still, immune responses against viral vectors play a crucial role in determining the long-term experimental and clinical effectiveness - and eventual fate - of viral vectors. To understand the phenomena responsible for the limitations imposed by the immune system on gene therapy, two approaches have been used. On the one hand, the direct consequences of injecting viral vectors into animals have been studied. These have determined that viral vectors induce the release of cytokines, interleukins, activate macrophages, induce T-cell and Bcell responses, induce viral-neutralizing antibodies, and induce the activation of the endothelium. ${ }^{1-6}$ Conversely, the role of many of these mechanisms has been tested in transgenic and knockout animals. These experiments have determined that the lack of complement, T cells, $B$ cells, interleukins, interferons, and various methods used to downmodulate immune responses (CTLA4Ig; sCD40L, etc) will prolong transgene expression in various tissues such as the liver, muscle, lung, or heart. ${ }^{7-9}$

However, it remains difficult to determine which mechanisms regulate transgene longevity and which are epiphenomena. ${ }^{1-4}$ Two recent issues in Gene Therapy Journals have been devoted to the mechanisms underlying immune responses to viral vectors. ${ }^{10,11}$ To identify the critical mechanisms, genetic approaches may provide the way forward by allowing a hypothesis-free search for those genes that direct the relevant immune responses to viral vectors.

In a very original paper published in this issue of Gene Therapy, Zhang et $a l^{12}$ have applied genetic technologies to discover regions of the genome that may have a causal relationship to the longevity of adenovirus-mediated transgene expression in the liver. Specifically, they sought to determine the existence of quantitative trait loci (QTL) that linked to the longevity of transgene expression in the liver, the presence of cytotoxic $\mathrm{T}$ lymphocytes (CTLs) against adenovirus, and serum cytokines. A QTL is a restricted region of the genome that plays a role in the quantitative variation of the trait being studied; it is analyzed by correlating sequence variations in the genome with variations in traits that vary quantitatively in the population under study. Once identified, the QTL is most likely a polymorphism affecting protein function or the levels of protein expression. ${ }^{13-15}$

To determine these QTL, they utilized 20 strains of recombinant inbred mice ${ }^{16-18}$ derived from a cross between C57BL/6J and DBA/2J; ${ }^{19}$ each recombinant inbred strain contains chromosomal regions that are derived from either parent, and they are homozygous at each locus (see Figure 1 for a schematic view of the production of recombinant inbred strains).

Liver $\beta$-galactosidase expression was measured for up to 50 days postinjection. At this stage, while some strains had lost $>90 \%$ of liver $\beta$-galactosidase expression, in others $\beta$-galactosidase expression was only reduced by $<50 \%$. Over the same time course, they measured parameters a priori linked to the longevity of transgene expression in the liver: serum levels IFN- $\gamma$, IL-6, and TNF- $\alpha$, and levels of anti-adenovirus (CTLs).

Correlations were established between $\beta$-galactosidase expression in the liver and individual parameters measured in the 20 different recombinant inbred strains. The authors found a very strong and statistically significant correlation of the expression of $\beta$-galactosidase and the level of anti-adenovirus CTLs, while there was no correlation between the liver expression of $\beta$-galactosidase and serum levels of IFN- $\gamma$, TNF- $\alpha$, or IL-6.

Statistical analysis was then used to compare the distribution of fairly evenly spaced chromosomal microsatellite markers throughout the genome of the strains studied, and each of the quantitative traits under evaluation (eg CTLs, cytokines, etc). Statistically significant QTLs represent areas of the genome whose presence correlates with the particular phenotype under consideration, for example, CTL. QTLs determined at long times post-virus administration reflect the capacity to clear $\beta$ galactosidase expression from the liver; this QTL mapped to Chr 15 at $42.8 \mathrm{cM}$, and Chr 19 at $33 \mathrm{cM}$. A QTL for CTL activity was identified on Chr 7 at $41 \mathrm{cM}$, and between 57.4 and 65.2, and on Chr 15 at $61.7 \mathrm{cM}$, and Chr X at $27.8 \mathrm{cM}$. QTLs for cytokine levels were also detected. While their levels correlated with each other, they did not correlate with either $\beta$ galactosidase clearance from the liver, or the presence of CTLs against adenovirus-infected cells.

We have learned from this paper that the host immune responses that regulate the longevity of adenovirusmediated transgene expression in the liver is a polygenetic trait. The genetic determinants of this response can be studied in a statistically stringent manner, that may determine such immune responses, particular regions of the genome that may determine such immune responses. Also, this paper demonstrates the existence of strong strain differences in transgene expression and immune responses, and suggests that this may also occur in other strains and species. The importance of this to clinical gene therapy, and as an 
Differential host genetic makeup leads to strain-dependent differences in host immune response and longevity of AdLacZ expression in the liver
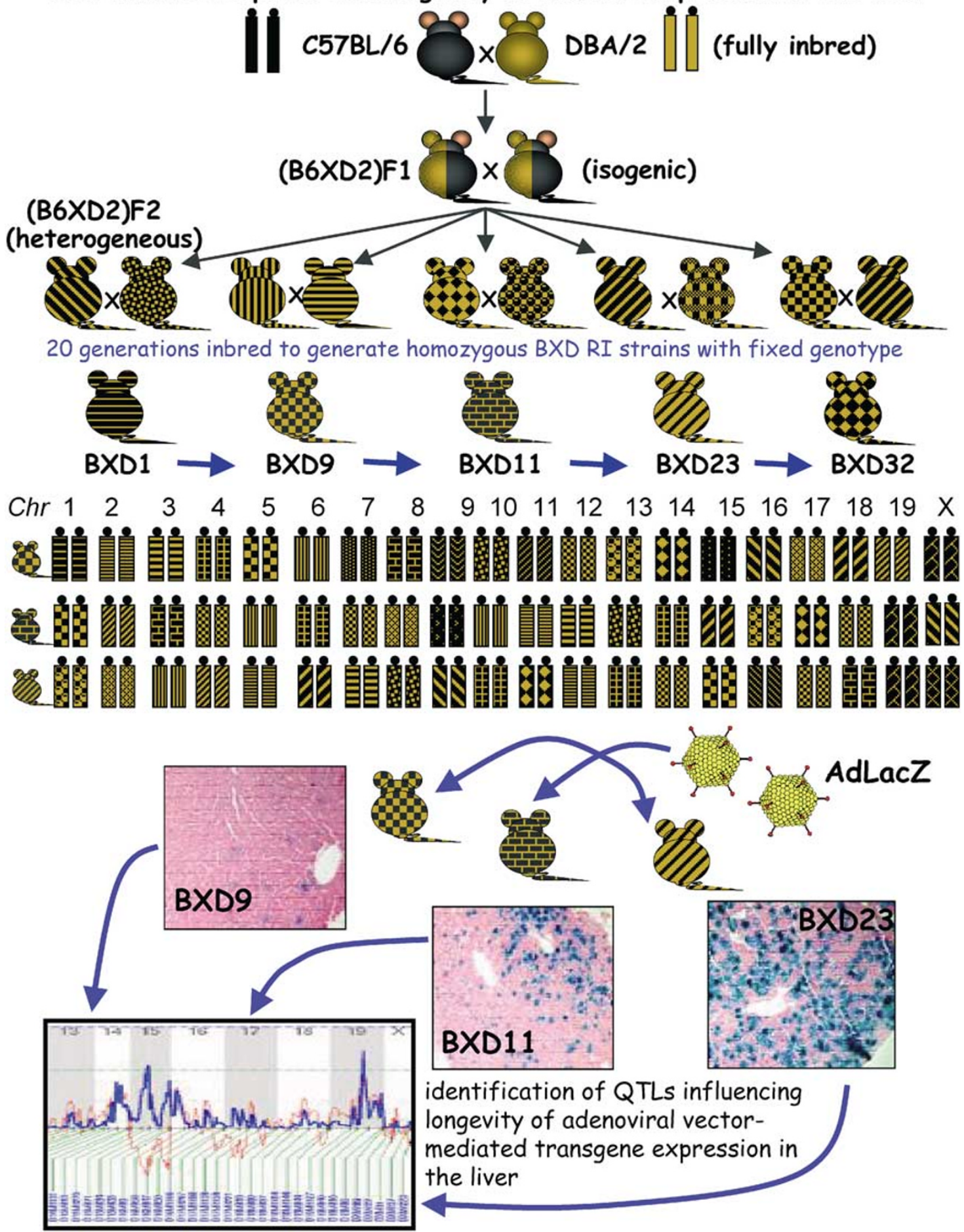

Figure 1 This figure summarizes the process involved in discovering QTLs determining anti-adenoviral immune responses eliminating vector-mediated $\beta$ galactosidase expression in the liver. The upper part of the figure illustrates how, from two inbred mouse strains, recombinant inbred strains (BXD1$B X D 23)$ are generated by intercrossing $F_{2}$ mice for 20 generations. Each of the recombinant inbred strains are homozygous at each locus, as illustrated schematically for three strains in the middle of the figure. The lower third of the figure illustrates how adenovirus is then injected into all BXD recombinant inbred strains, and B-galactosidase expression, CTL levels, and cytokines are then measured. The correlation among different measurements is then performed and analyzed statistically. Longevity of transgene expression in the liver was only statistically correlated with levels of CTL. Genetic markers that are approximately evenly distributed throughout the mouse genome are then used to determine the variability of the genetic structure of each strain and compared to the variability of each measured response in the series of recombinant inbred mouse strains, and the results are then analyzed statistically. Statistically significant values indicate the location of potential QTL. Statistically significant QTL were identified for $\beta$-galactosidase expression, the level of CTLs, IFN- $\gamma, T N F-\alpha$, and IL-6. 
experimental model in which to test this variability is very high.

The next step will consist of identifying the sequence variations in the genome that contribute to each QTL. In spite of many recent advances in mouse genomic studies, this is a difficult task. One advantage of using the BXD recombinant inbred strains of mice is that the Mouse Genome Project will provide the complete sequence of the parental B6 and D2 genomes to interested investigators in the near future. Given that many of the polymorphic genes between B6 and D2 mice have been identified, the sequence of the $B X D$ recombinant inbred strains will essentially be known. In the long run, the search aims to identify either a nucleotide polymorphism within the coding region that determines protein function, or a polymorphism within the promoter regions that affect the level of gene expression. ${ }^{13-18}$

The power of the studies published is also necessarily limited to the QTLs defined a priori. Alternative hypotheses could propose, for example, that IFN- $\gamma$ released by subtypes of immune cells directly in the liver contributes to clearing of adenovirus from the liver. Since these hypotheses have not been proposed, even though any of these may be of great importance, they have not been addressed in this manuscript. Importantly though, the same set of 20 recombinant inbred strains used in this work are available for the testing of any further hypothesis concerning the mechanisms of immune mechanisms that affect adenovirus vectormediated transgene expression.

In summary, this paper represents an important advance in our capacity to examine and understand the molecular and genetic basis of the immune mechanisms that regulate viral vector-mediated transgene expression. To do so, much future hard work awaits its authors in narrowing down the QTL to a unique molecular basis. The possibility of identifying those genes and their sequence variations that determine either the longevity of $\beta$-galactosidase expression or anti-adenoviral CTLs remains of great importance and general relevance to Gene Therapy.

\section{Acknowledgements}

I thank John Mountz for many challenging discussions, and joint preparation of Figure 1.

$P R$ Lowenstein is at the Gene Therapeutics Research Institute, Cedars-Sinai Medical Center, Davis Bldg Room R5090, Beverly Blvd 8700, USA; and Department of Medicine, David Geffen School of Medicine, University of California Los Angeles, Los Angeles, CA, USA. PR Lowenstein is Bram and Elaine Goldsmith Chair in Gene Therapeutics. The support of the Board of Governors is kindly acknowledged. Work in my laboratory is funded by the National Institutes of Health. E-mail: lowensteinp@cshs.org

1 Yang Y, Wilson JM. Clearance of adenovirus-infected hepatocytes by MHC class I-restricted CD4+ CTLs in vivo. J Immunol 1995; 155: 2564-2570.

2 Yang Y, Ertl HC, Wilson JM. MHC class I-restricted cytotoxic $\mathrm{T}$ lymphocytes to viral antigens destroy hepatocytes in mice infected with E1-deleted recombinant adenoviruses. Immunity 1994; 1: 433-442.

3 Yang $Y$ et al. Immune responses to viral antigens versus transgene product in the elimination of recombinant adenovirusinfected hepatocytes in vivo. Gene Therapy 1996; 3: 137-144.

4 Yang Y, Li Q, Ertl HC, Wilson JM. Cellular and humoral immune responses to viral antigens create barriers to lung-directed gene therapy with recombinant adenoviruses. J Virol 1995; 69: 2004-2015.

5 Chirmule $\mathrm{N}$ et al. Immune responses to adenovirus and adeno-associated virus in humans. Gene Therapy 1999; 6: 15741583.

6 Molinier-Frenkel $\mathrm{V}$ et al. Immune response to recombinant adenovirus in humans: capsid components from viral input are targets for vector-specific cytotoxic T lymphocytes. J Virol 2000; 74: 7678-7682.
7 Kafri T et al. Cellular immune response to adenoviral vector infected cells does not require de novo viral gene expression: implications for gene therapy. Proc Natl Acad Sci USA 1998; 95: 11377-11382.

8 Amalfitano A, Parks RJ. Separating fact from fiction: assessing the potential of modified adenovirus vectors for use in human gene therapy. Curr Gene Ther 2002; 2: 111-133.

9 Benihoud $\mathrm{K}$ et al. Efficient, repeated adenovirus-mediated gene transfer in mice lacking both tumor necrosis factor alpha and lymphotoxin alpha. J Virol 1998; 72: 9514-9525.

10 Anderson WR. Adenoviral vector safety and toxicity. Hum Gene Ther 2002; 13: 1175.

11 Lowenstein PR. Immune responses to viral vectors for gene therapy. Gene Therapy 2003; 10: 933-998.

12 Zhang HG et al. Identification of multiple genetic loci that regulate adenovirus gene therapy. Gene Therapy 2003; 11: 4-14.

13 Moore KJ, Nagle DL. Complex trait analysis in the mouse: the strengths, the limitations and the promise yet to come. Annu Rev Genet 2000; 34: 653-686.

14 Doerge RW. Mapping and analysis of quantitative trait loci in experimental populations. Nat Rev Genet 2002; 3: 43 52.

15 Flint J, Mott R. Finding the molecular basis of quantitative traits: successes and pitfalls. Nat Rev Genet 2001; 2: 437-445.

16 Williams RW, Gu J, Qi S, Lu L. The genetic structure of recombinant inbred mice: high-resolution consensus maps for complex trait analysis. Genome Biol 2001; 2: 1-18.

17 Threadgill DW, Hunter KW, Williams RW. Genetic dissection of complex and quantitative traits: from fantasy to reality via a community effort. Mamm Genome 2002; 13: 175-178.

18 Markel $\mathrm{P}$ et al. Theoretical and empirical issues for marker-assisted breeding of congenic mouse strains. Nat Genet 1997; 17: 280-284.

19 Plomin R, McClearn GE, Gora-Maslak G, Neiderhiser JM. Use of recombinant inbred strains to detect quantitative trait loci associated with behavior. Behav Genet 1991; 21: 99-116. 